\title{
RÉFLEXIONS SUR UNE MÉTHODE D'ÉTUDES DES RELATIONS ENTRE FACTEURS ÉCOLOGIQUES ET CARACTÉRISTIQUES DES PEUPLEMENTS
}

\author{
J. GARBAYE, Ph. LEROY (1), F. LE TACON et G. LEVY \\ Station de Recherches sur les Sols forestiers et la Fertilisation, \\ Centre national de Recherches forestières, 54 - Nancy \\ Institut national de la Recherche agronomique
}

\section{RESUME}

Dans le but d'étudier l'action des facteurs du milieu et essentiellement du sol sur la production ou toute autre caractéristique des peuplements, nous décrivons une méthode générale de travail, inspirée des très nombreux travaux effectués depuis près de cinquante ans sur ces problèmes.

Il s'agit tout d'abord de choisir dans une région climatiquement aussi homogène que possible, un certain nombre de points d'étude couvrant la gamme complète de sols ou de stations.

En chaque point, nous effectuons la mesure des caractéristiques du peuplement proprement dit, la mesure des caractéristiques de nutrition et enfin la mesure des caractéristiques du milieu et en particulier du sol.

Nous essayons ensuite de rechercher les liaisons entre ces trois séries de données par deux types de procédés.

Nous pouvons utiliser des procédés très simples, graphiques ou statistiques, faisant intervenir soit des facteurs isolés mais importants, soit des notions synthétiques telles que le type génétique de sol ou le type de roche-mère.

Nous pouvons également utiliser des procédés beaucoup plus complexes tels que les analyses multivariates.

Nous pouvons ainsi espérer pouvoir prévoir la production en fonction des facteurs du milieu et également acquérir des bases solides pour la mise en place d'expériences d'amélioration des conditions de milieu.

Les résultats obtenus seront également le point de départ d'un certain nombre de recherches beaucoup plus fines.

Enfin, ce type d'étude nécessite la collaboration de la plupart des disciplines de la Recherche forestière.

(1) Actuellement détaché au C.R.P.F. Lorraine-Alsace, 57 - Metz. 


\section{INTRODUCTION}

Nous ne connaissons actuellement que les très grandes lignes des exigences des essences indigènes ou introduites vis-à-vis des facteurs du milieu.

L'action de ces facteurs du milieu sur la production nous échappe partiellement. Ce manque de connaissance nous laisse souvent dans l'embarras quand il s'agit de déterminer la meilleure façon de mettre en valeur un fonds forestier.

Dans le cadre d'une politique forestière dynamique, il faut mettre en œuvre des moyens de recherches susceptibles de fournir des données certaines et facilement utilisables sur le choix des essences ou la prévision de la production en fonction des caractéristiques du milieu.

En s'appuyant sur ces données, le sylviculteur pourra chercher à optimiser la production en agissant dans deux directions :

- choix des essences les mieux adaptées aux diverses conditions de station,

- modification de certains facteurs du milieu limitant la croissance.

Néanmoins, la notion de choix du matériel végétal à utiliser aura toujours une résonance toute particulière en matière forestière. En effet, la longueur des révolutions ainsi que la faiblesse des revenus tirés de la production de bois limitent considérablement les possibilités d'investissements destinés à modifier le milieu. Dans ce contexte, le choix d'une essence bien adaptée aux conditions de station peut réduire le coût des interventions sur le milieu à un niveau compatible avec les nécessités de la rentabilité. Il faut toutefois se garder de minimiser l'intérêt des interventions sur les facteurs du milieu. Il suffit de penser aux gains de production acquis au moyen de la fertilisation, notamment sur peuplement adulte, pour constater que des investissements faibles conduisent parfois à des bénéfices importants.

Soulignons que le choix du matériel végétal présente souvent pour le forestier un caractère critique. En ce domaine, une erreur est plus grave qu'en agriculture, car elle se répercute d’année en année pendant toute la vie du peuplement.

Comment aborder ce problème du choix de l'essence, de prévision de production et d'amélioration du milieu en fonction des caractéristiques de station ?

Deux possibilités nous sont offertes: l'expérimentation et l'enquête.

L'expérimentation est la méthode la pius logique, mais la plus longue et la plus coûteuse. Elle est la seule possible pour les essences d'introduction récente qui ne constituent pas encore des peuplements adultes.

Pour les essences indigènes ou les essences exotiques introduites de longue date, il est préférable d'utiliser la seconde méthode qui offre l'avantage de la rapidité. On ne peut en effet attendre des dizaines d'années avant d'avoir des résultats susceptibles de donner aux sylviculteurs un outil de travail sûr et efficace.

\section{I. - EVOLUTION DES IDEES CONCERNANT LES METHODES D'ETUDE DES RELATIONS MILIEU-PRODUCTION}

Depuis longtemps, les forestiers ont cherché à déterminer dans quelle mesure le milieu pouvait avoir une influence sur le comportement des peuplements. Le problème avait déjà été posé au XVIII" siècle, mais il faut attendre le début du $x^{e}$ siècle pour 
que de véritables tentatives soient effectuées. Les premiers essais ont été effectués sous un angle non explicatif. Il s'agissait uniquement de montrer les « relations » pouvant exister entre la présence de certaines plantes harbacées dites indicatrices et la production (Korstian, 1917 et 1919). Cette méthode floristique, particulièrement intéressante dans son application en raison de la facilité d'identification des plantes herbacées indicatrices, a été reprise depuis à maintes reprises et considérablement améliorée (RIGG, 1929 ; Hartmann, 1926 ; Cajander, 1926 ; Colle, 1928 ; Dagnelie, 1956, etc.).

Avec DAGNelıe apparaît une tendance qui consiste à allier à la méthode floristique proprement dite une étude véritable de l'influence des facteurs du milieu.

Ce type de méthode mixte à la fois floristique et écologique a été largement employé et amélioré. En France, citons les études de Duchaufour, Debazac, Jacamon, Parde en forêt du Ban d'Etival, puis de Duchaufour, Bonneau, Debazac, Parde en Argonne; dans ce type d'étude, les facteurs écologiques étaient étudiés globalement et synthétisés par le type de sol. Mais l'action des facteurs particuliers du milieu n'était pas envisagée. Plus récemment, Aussenac et Becker, puis Becker, sur le Hêtre, ont utilisé cette méthode mixte en développant les moyens de traitement des données floristiques et en essayant de faire apparaître les facteurs du milieu, non pas uniquement d'une manière synthétique, mais en tant que tels.

Si l'on exclut l'aspect floristique, on rejoint par-là une autre méthode d'aborder le problème et que l'on peut qualifier de directe. Cette méthode directe s'est développée parallèlement à la méthode floristique pure ou à la méthode mixte. Elle consiste à rechercher les liaisons existant entre la production ou un indice de production et les facteurs du milieu sans passer par la flore herbacée.

IlvesAllo en a défini le principe et a proposé une méthode de travail en 1923. Mais c'est surtout après 1940 que les études se sont multipliées, essentiellement aux Etats-Unis. Citons Carmean, Coile, Colle et Schumacker, Donahue, Gaiser, Gessel, Gessel et Lloyd, Lunt, Stoeckeller, Tamm et Tarrant, Viro, YoungBerg, Scholz, etc. En France, Decourt, Godron et Tomassone ont travaillé en Sologne sur le Pin sylvestre. Ces derniers autzurs ont abordé le problème avec des moyens mathématiques modernes. OswaLd a travaillé dans ce sens en Arłèche, mais en tenant compte de facteurs autres que le sol.

Ces dernières années, cette méthode d'étude directe a été notablement améliorée par l'utilisation des techniques d'analyse foliaire. L'analyse foliaire mise au point en agriculture fait vraiment partie des techniques d'investigation forestière depuis une quinzaine d'années environ, et peut rendre de très grands services.

Dans cet article, nous allons essayer de faire le point sur les possibilités d'investigation qui sont à notre disposition pour tenter de comprendre l'influence des facteurs du milieu, et plus particulièrement du sol, sur les caractéristiques des peuplements.

Nous excluons ici les procédés indirects basés sur la floristique, et d'autre part nous n'insisterons aucunement sur l'aspect dendrométrique.

\section{II. - METHODE GENERALE DE TRAVAIL}

La méthode d'enquête que nous utilisons implique :

$1^{\circ}$ la sélection des régions où la recherche s'avère prioritaire ;

$2^{\circ}$ la délimitation dans ces régions des zones de travail ;

$3^{\circ}$ le choix d'un système d'échantillonnage ; 
$4^{\circ}$ la détermination de la nature des mesures effectuées aux points d'étude ;

$5^{\circ}$ le choix d'une ou de plusieurs méthodes d'interprétation.

Pour le premier point, l'initiative du choix échappe en partie à la compétence directe du chercheur, nous ne ferons que le citer.

\section{1. - Délimitation de la zone de travail.}

Il faut tout d’abord tenir compte des caractéristiques climatiques qui déterminent en grande partie les potentialités de production d'une station. Or, les facteurs du climat sont extrêment difficiles à mesurer et variables d'une année à l'autre. Si un réseau de stations météorologiques dense installé dans la région d'enquête ne fournit pas des informations complètes, depuis un grand nombre d'années, il est préférable d'essayer d'éliminer l'influence climatique en choisissant une zone de travail climatiquement homogène ou aussi peu variable que possible.

L'homogénéité par rapport aux principales caractéristiques climatiques sera d'autant plus grande, que la surface de la zone de travail sera plus petite.

Néanmoins, des variations liées à des facteurs microclimatiques subsisteront toujours et devront être prises en considération d'une manière ou d'une autre.

Dans un premier temps les enquếes seront ainsi limitées à des zones climatiquement homogènes. On pourra ensuite travailler de proche en proche, comparer les zones entre elles et éventuellement faire des regroupements ou de nouveaux découpages.

Il n'est pas exclu dans l'avenir de pouvoir généraliser les résultats obtenus sur un certain nombre de zones de travail, à un ensemble de régions beaucoup plus vaste. Cette généralisation devra être prudente et respecter les conditions suivantes: enquêtes ;

- elle ne portera que sur des essences ou groupes d'essences étudiées au cours des

- les régions où seront utilisés les résultats établis dans les zones d'enquêtes ne devront pas être trop éloignées écologiquement de ces dernières.

Il est cependant possible de choisir parfois une zone climatiquement hétérogène à condition de pouvoir lier les variations à une cause bien déterminée. C'est, par exemple, le cas des variations de climat provoquées par des changements d'altitude (OSwALD, 1968).

\section{2. - Choix des points d'étude.}

Il s'agit d'abord de rechercher à l'intérieur de la zone choisie toutes les essences qui y sont utilisées en éliminant celles qui, à priori, n'ont manifestement aucun intérêt économique.

Pour chacune des essences retenues, il faut ensuite sélectionner un certain nombre de points d'échantillonnage, de manière à couvrir à la fois la fourchette complète de production et la gamme des sols ou des stations de la zone de travail.

Ce choix des points d'étude dans une zone de travail implique deux impératifs:

- une bonne connaissance des sols, de leur fréquence et de leur répartition, Connaissance qui peut être acquise dans l'idéal par cartographie à petite ou moyenne échelle $(1 / 100.000$, par exemple) ;

- la possession d'un inventaire quasi complet des peuplements adultes. Pour chaque peuplement on déterminera approximativement l'âge des arbres, leur vitesse de croissance et le type de sol (sondages à la tarière pédologique et à la tarière de Pressler).

Ce travail d'approche permet le choix des points d'étude avec une probabilité de couvrir l'ensemble des facteurs de variations. De cette façon on a une chance de pouvoir expliquer logiquement les différences de production qui ont pu être relevées.

Le nombre des points d'étude doit être assez élevé à la fois pour aboutir à des conclusions solides et pour utiliser toutes les possibilités des techniques statistiques.

\section{3. - Nature des mesures effectuées aux points d'étude.}

Chaque point d'étude fera l'objet de trois catégories essentielles de mesures ou quatre éventuellement.

a) la mesure des caractéristiques des peuplements (production, composante de la production, qualité technologique) ; 
b) la mesure des facteurs du milieu ou variables explicatives. Ces facteurs seront essentiellement d'ordre pédologique et microclimatique, puisque au départ la variabilité macroclimatique a été éliminée par le choix des zones de travail;

c) la mesure de facteurs que l'on pourrait qualifier de mixtes car appartenant à la fois au végétal et au milieu. Ce sont les analyses foliaires effectuées soit sur l'essence étudiée soit sur la flore herbacée, si on veut aborder le problème de manière indirecte ;

d) le relevé de la flore herbacée devra d'ailleurs constituer chaque fois que cela sera possible la quatrième série de mesures.

Dans ce cas on abordera les liaisons milieu caractéristiques des peuplements de manière indirecte. On compliquera le problème sans d'ailleurs changer le principe de la méthode que nous exposons.

Dans ce qui va suivre nous allons nous limiter pour des raisons de clarté à certains aspects du problème.

\section{III. - MESURE DES CARACTERISTIQUES DU PEUPLEMENT}

(Variables à expliquer)

La caractéristique la plus importante d'un peuplement est sa production de bois, dont dépend le plus souvent l'essentiel du revenu argent. Ce revenu argent dépend aussi, et dans quelques cas beaucoup, de la qualité des produits récoltés ; mais, dans un but de simplification, nous ne raisonnerons ici que sur les caractéristiques de production proprement dites.

Le raisonnement que nous allons développer est parfaitement applicable dans le cas où nous nous intéresserions aux caractéristiques technologiques dépendant du milieu.

Il faut donc, puisque nous avons décidé de nous limiter à la production proprement dite, connaître cette production en chacun des points d'étude.

Nous pouvons l'apprécier directement par l'installation de placettes temporaires (Decourt, 1966-1967).

Ce système de placettes utilisées pour la construction de tables de production (DeCourt) a un inconvénient pour le type d'étude par enquête qui nous occupe.

L'installation d'une placette nécessite en effet une surface suffisamment grande (30 à 40 ares), ce qui entraîne des risques d'hétérogénéité du sol ; l'influence des facteurs édaphiques devient alors difficile à déterminer avec précision.

Pour ce type d'étude par enquête, il est nécessaire de travailler sur la plus petite surface possible compatible avec la mesure d'un indice de production, de façon à éliminer au maximum les risques d'hétérogénéité du sol.

Il est donc préférable d'utiliser des placettes ponctuelles (3 à 5 ares) permettant d'avoir accès indirectement à la production par la mesure d'un indice, comme l'a fait Decourt pour le Pin sylvestre en Sologne.

La hauteur dominante, facile à mesurer, est un excellent indice de production, dont la valeur est admise par la majorité des stations de sylviculture.

Il est cependant intéressant de relier la hauteur dominante à la production dans la région considérée à l'aide des tables de production. Si de telles tables n'existent pas, il faut s'appuyer sur un certain nombre de placettes de production.

Un indice de production basé sur la hauteur dominante est pourtant suffisant pour établir un classement relatif inter-station. Mais en se limitant à cet indics, il faut éliminer l'infuence de l'âge; en effet, il est souvent impossible de travailler sur des 
peuplements d'âge identique, ou sur de très vieux peuplements dont la croissance en hauteur a atteint un palier.

Pour des peuplements d'âge divers, l'analyse de tiges permet :

1) de reconstituer l'évolution de la hauteur dominante dans le temps;

2) de comparer les hauteurs à âge constant, comme l'a fait Oswald en haute Ardèche ;

3) de raisonner sur les caractéristiques mathématiques des courbes de croissance.

Il peut être en outre intéressant de raisonner non pas sur la résultante de la croissance (production d'un volume), mais sur les composantes (croissance en hauteur, croissance en diamètre, masse foliaire, poids et longueur des feuilles ou des aiguilles, etc.), qui sont évidemment liées entre elles, mais dont le déterminisme peut être différent.

L'étude de ces déterminismes peut apporter des conclusions partielles aidant à la compréhension de phénomènes plus généraux.

\section{IV. - MESURES DES CARACTERISTIQUES DU MILIEU OU VARIABLES EXPLICATIVES}

\section{Variables microclimatiques}

Les variations de climat général ayant été éliminées, en principe seules interviendront les variables microclimatiques.

Il est bien certain que l'on ne pourra les esiimer qu'approximativement avec des méthodes indirectes. Il est hors de question de pouvoir les mesurer directement de manière systématique.

Une approche satisfaisante pourra être obtenue par la mesure de certains facteurs tels que les variations d'altitude, la position sur la pente, le type de pente, le degré de pente ou l'exposition.

\section{Variables du sol}

Si l’on fait abstraction de la variabilité génétique, les caractéristiques du sol constituent en zone climatiquement homogène l'essentiel des explications des variations de production, et nous nous attacherons donc à la mesure de toutes ces caractéristiques.

Nous pouvons, en premier lieu, utiliser des variables synthátiques. Ce sera essentiellement le type génétique de sol, le type d'humus et la roche-mère.

Il est évidemment nécessaire d'aller plus loin que ces notions globales et de mesurer individuellement le plus grand nombre possible de caractéristiques physiques et chimiques du sol.

Pour faciliter le travail, on pourra dans certaines zones d'étude classer d'abord les points en fonction de ces variables synthétiques. Pour tous les points d'un même groupe, l'étude des facteurs explicatifs sera identique ; en plus des mesures communes à tous les points quelle que soit la nature du sol, on étudiera plus en détail dans chaque groupe les facteurs qui déterminent l'originalité de ces groupes, par exemple les nappes 
dans les sols hydromorphes (profondeur, durée d'engorgement, potentiel d'oxydoréduction, etc.).

Nous possédons tout un arsenal de techniques permettant d'estimer, avec une certaine approximation, il est vrai, les principales caractéristiques physiques et chimiques des sols.

Caractéristiques physiques:

- capacité maximale de rétention en eau utile,

- densité apparente,

- micro et macroporosité,

- stabilité structurale,

- perméabilité,

- compacité,

- pourcentage de terre fine,

- volume prospecté par les racines,

- granulométrie.

Caractéristiques chimiques:

- teneur en azote total,

- teneur globale en matière organique,

- pourcentages des différentes fractions de matières organiques,

- capacité totale d'échange,

- taux de saturation,

- éléments échangeables, semi-totaux et totaux,

- $\mathrm{pH}$,

- teneur en calcaire actif et total,

- teneur en phosphore assimilable, semi-total et total,

- aluminium libre et échangeable,

- oligo-éléments, etc.

Les déterminations que l'on peut effectuer sur un sol sont extrêmement nombreuses, mais leur intérêt est variable. Nous nous attacherons, pour notre problème, à déterminer seulement les caractéristiques dont l'influence s'est révélée prépondérante sur la croissance des végétaux.

Ces techniques d'analyses sont rodées et progressent constamment. Néanmoins, de multiples problèmes demeurent. Les résultats obtenus n'ont que valeur de comparaison et ne se rapprochent que plus ou moins de la réalité.

De plus, certaines variables du milieu sont extrêmement difficiles à mesurer, au moins rapidement et sur un grand nombre d'échantillons.

Nous nous trouvons donc en présence de deux types de difficultés: certaines techniques ne permettent qu'une évaluation approximative, et certains facteurs ne peuvent pratiquement pas être mesurés.

Voyons d'abord le premier type de problème.

Techniques ne donnant qu'une certaine évaluation des variables du milieu.

Certaines caractéristiques chimiques sont difficiles à déterminer en soi. C'est par exemple le cas du calcaire. La teneur en calcaire totale ne renseigne qu'imparfaitement 
sur le degré d'activité chimique du calcaire. Seules les pellicules externes des particules de calcaire ont une activité chimique dans le sol (calcaire actif).

On a donc essayé de chiffrer cette activité par action sur l'oxalate d'ammonium. Malheureusement, la matière organique vient perturber cette détermination et il est très long d'éliminer cette interaction. De même, dans le dosage du calcaire actif, le calcium échangeable réagit sur l'oxalate, ce qui donne des valeurs par excès.

Comme le dosage du calcium échangeable est difficilement réalisable en sol carbonaté, le dosage du calcaire actif devient problématique pour des teneurs inférieures à $3 \%$.

D'autres caractéristiques chimiques sont par contre facilement mesurables, mais nous ne connaissons pas exactement quelles formes sont absorbables par le végétal.

Ceci est particulièrement vrai pour le potassium et surtout le phosphore. Le phosphore constitue toujours un problème redoutable pour les agronomes et les forestiers. Les réactifs d'extraction sont multiples. L'idéal serait de trouver pour chaque groupe végétal et chaque grand groupe de sols des réactifs d'extraction adaptés, c'est-à-dire extrayant les formes du phosphore absorbées réellement par le végétal étudié.

Heureusement, des analyses foliaires viennent compléter les analyses de sols et pallient certaines de leurs insuffisances. Ces analyses foliaires fournissent en effet un indice lié à ce que l'arbre a réellement absorbé, et par conséquent donnent des informations sur la disponibilité réelle des divers éléments dans le sol. Nous pouvons même ensuite rectifier la méthode d'extraction du sol en fonction des résultats de l'analyse foliaire effectuée soit sur l'essence étudiée, soit dans certains cas, sur la plante herbacée.

\section{Variables difficilement mesurables.}

Si nous pouvons, pour la plupart des variables, arriver à une évaluation plus ou moins bonne, il existe deux ensembles de variables du milieu qui posent des problèmes extrêmement difficiles pour le type de travail que nous entreprenons: ce sont l'eau et l'azote.

Ces deux facteurs posent de tels problèmes essentiellement parce que, tout en appartenant aux facteurs du sol et absorbés par le végétal dans le sol, ils dépendent atissi des facteurs climatiques.

Ils dépendent évidemment du climat général, mais nous pouvons éliminer cette influence en travaillant en zone climatiquement homogène, comme nous l'avons vu. Cependant, des différences climatiques d'une année à l'autre garderont une influence dans cette zone pour les disponibilités en eau et en azote.

Il est extrêmement difficile de chiffrer un facteur qui varie au cours des saisons et dont les variations ne se répètent pas identiquement à elles-mêmes d'une année à l'autre.

Voyons d'abord le problème de l'eau. Nous pouvons déterminer la capacité maximale de rétention en eau utile. C'est évidemment un facteur important, mais insuffisant. Pour une même valeur de capacité de rétention en eau utile, et dans des conditions climatiques identiques, la disponibilité de l'eau peut être très différente pour deux sols suivant la saison, si l'un bénéficie d'apports latéraux d'eau par ruissellement et l'autre pas. De même, la présence d'une nappe temporaire ou permanente vient totalement bouleverser les données. 
Il est toujours possible d'établir des profils hydriques pendant une année ou plusieurs années consécutives, mais, en raison de la multiplicité des observations nécessaires à l'établissement de tels profils, il est impossible de l'effectuer sur toutes les placettes utilisées dans une enquête.

Par impossibilité matérielle, nous perdons obligatoirement une partie de l'information, et donc une partie de l'explication des variations de production.

Le problème de l'azote est tout aussi ardu et, dans un certain sens, plus complexe.

La production d'azote minéral d'un sol dépend des caractéristiques climatiques de l'année, de l'époque de l'année, des autres facteurs du sol, physiques et chimiques, des végétaux supportés par le sol, de la densité des peuplements, etc. De plus, l'apport direct par les eaux de pluie est loin d'être négligeable.

Dans ces conditions, la mesure d'un indice simple est insuffisante.

Le dosage de l'azote total du sol couplé avec celui de la matière organique (rapport $\mathrm{C} / \mathrm{N}$ ) apporte des informations assez grossières.

Pour avoir une bonne évaluation de la production azotée d'un sol, il est nécessaire de mettre en place un système de lysimètres, ce qui nécessite un nombre très important de mesures périodiques, ou, à défaut, d'effectuer uniquement des prélèvements périodiques in situ (BONNEAU, 1967).

Il existe un test qui permet une certaine évaluation de la production azotée et qui peut nous être d'un précieux secours. Il consiste à déterminer la quantité d'azote ammoniacal et nitrique produit par un échantillon pendant 6 semaines à $28^{\circ}$. Cette production est plus oli inoins équivalente à cəlle d'un sol en place pendant une année. En fait, il est préférable de considérer cette production comme un indice d'activité propre à l'humus.

Enfin, nous disposons, comme pour les autres éléments, d'un test indirect, celui de lanalyse foliaire. Il samble d'ailleurs que dans cartains cas l'analyse foliaire d'une mlante herb ćé à grande amplitude écologique pourrait être très intéressante et nous fournir des renseignements sur le potentiel de production azotée des stations indépendamment de l'essence forestière présente.

Mais, de toute façon, quelles que soient les méthodes utilisées seules ou simultanément, ce problème d'évaluation de la capacité de production azotée d'une station est particulièrement difficile à résoudre dans le cas d'une étude par enquête, où le nombre de stations étudiées doit être grand.

\section{V. - DETERMINATION DES RELATIONS SOL PRODUCTION NUTRITION PREVISION DE LA PRODUCTION}

Nous sommes maintenant en présence de trois séries de variables:

- des variables à expliquer caractéristiques du peuplement ;

- des variables explicatives caractéristiques du milieu;

- des variables mixtes, essentiellement des caractéristiques de nutrition, obtenues à partir des analyses foliaires. Ce ne sont pas des variables explicatives vraies puisque n'appartenant pas au milieu. Elles sont la résultante des caractéristiques chimiques et physiques du sol et de certaines caractéristiques physiologiques de l'arbre. Nous pourrons les traiter comme variables explicatives dans le cas de l'étude de l'influence de 
la nutrition sur la production. Elles deviendront des variables à expliquer lorsque nous tenterons de les relier aux caractéristiques du milieu.

\section{Méthodes d'interprétation complexes}

Ces trois séries de variables constituent un ensemble très complexe, d'autant plus qu'elles sont liées entre elles par des biais biologiques.

Jusqu'à présent, les difficultés d'interprétation étaient énormes et les résultats que l'on pouvait espérer obtenir étaient assez minces.

Or, depuis quelques années, des techniques statistiques dites analyses multivariates ont été mises au point pour le traitement de problèmes où plusieurs facteurs plus ou moins liés entre eux agissent simultanément.

En France, ces techniques ont été introduites en recherche forestière par TomasSONE et Millier et commencent à être largement utilisées; ce sont l'analyse des composantes principales, l'analyse factorielle, l'analyse par régression progressive et l'analyse par régression orthogonale. Ces deux dernières analyses permettent d'estimer une variable à expliquer (ici la production), soit en fonction de variables explicatives simples mais liées entre elles (ici les facteurs du milieu), soit en fonction de variables composites et nouvelles, les composantes principales indépendantes entre elles. Ces diverses analyses ont été décrites par Tomassone (1966-1967-1968).

Nous voyons tout l'intérêt de ces techniques dans le problème qui nous intéresse. Nous pouvons en effet espérer pouvoir estimer la production ou un indice lié à la production en fonction des caractéristiques du milieu. Si l'estimation est suffisante, nous pourrons ensuite mesurer les caractéristiques du milieu qui ont le rôle le plus important dans l'explication de la production, et arriver ainsi à une prévision de cette production.

Nous pensons également, par de telles méthodes, obtenir des informations très importantes sur la manière dont peuvent agir certains facteurs du milieu sur la croissance et sur la manière dont ces facteurs peuvent agir les uns sur les autres.

Nous espérons ainsi avoir accès à une information qu'il est impossible d'obtenir par toute autre méthode. La plupart du temps, en effet, les biologistes, devant la complexité du problème, se placent dans des conditions telles que tous les facteurs soient constants, sauf un ou deux que l'on veut justement étudier. Un tel type d'étude fournit évidemment des résultats que l'on ne pourrait obtenir autrement, mais il en résulte une perte d'information certaine et une vue partielle du problème.

Inversement, une étude globale telle que nous l'envisageons apportera des éléments nouveaux impossibles à obtenir autrement, mais également des résultats limités en raison de la complexité du milieu et de l'imperfection des méthodes de mesure et des méthodes d'interprétation.

De toute façon, une fois l'enquête terminée, l'installation d'un certain nombre d'expérimentations est indispensable, afin de confirmer ou d'infirmer une partie des résultats obtenus.

\section{Méthodes d'interprétation simples}

Pour traiter un tel problème, il est bien évident que sans des techniques mathématiques appropriées, il serait difficile d'obtenir des résultats, et même assez illusoire 
de vouloir entreprendre un tel travail d'investigation sans des méthodes d'analyse relativement fines.

Il n'est cependant pas impossible d'arriver à des résultats intéressants par des procédés très simples.

Les propriétés d'un sol peuvent être globalement caractérisées par le type génétique et la roche-mère, ou le type d'humus, par exemple. D'autres notions synthétiques, comme les groupes écologiques de végétaux herbacés, peuvent être utilisés. Nous citerons l'étude du Ban d'Etival (Duchaufour et al., 1958) qui permet de donner des fourchettes de production en fonction des types de sols et des groupements floristiques correspondants pour le Sapin pectiné et le Pin sylvestre.

Dagnelie a mis en évidence pour le Hêtre une relation entre la productivité et cerrtaines associations de plantes herbacées. La production du Chêne dans l'ouest de la France dépend du degré d'évolution des profils de sols; les productions les plus basses sont observées sur sols podzoliques (LERoy, 1967-1968). Des observations semblables ont été effectuées sur le Hêtre dans le centre de la France (BEcker, 1968). Le dépérissement du Pin maritime dans les Landes de Gascogne peut s'expliquer globalement par le type génétique de sol (Bonneau, Gelpe, Le TaCon). On pourrait ainsi multiplier les exemples.

Il est donc possible de donner des fourchettes de variation de la production en fonction de ces notions synthétiques.

Il est d'autre part possible qu'à l'intérieur de certains groupes de sols la production soit très directement liée à des critères simples. On peut alors, à partir de ces critères simples, prévoir facilement la production à l'intérieur de ces groupes.

Il est difficile d'aller plus loin par ce procédé très simple, car on se heurte immédiatement à l'interaction entre variables du milieu.

Si l'on veut améliorer la précision de ces fourchettes, il est absolument indispensable de recourir aux procédés d'investigations complexes d'analyses multivariates.

\section{VI. - PROLONGEMENTS D'UNE TELLE ETUDE}

Le premier objectif que nous nous sommes fixés en utilisant une telle méthode de travail est l'acquisition d'informations nous permettant de déterminer l'essence qui assurera la meilleure production dans des conditions de milieu données, et de chiffrer cette production potentielle avec le plus de précision possible, uniquement en fonction de certains facteurs du milieu.

Mais il est bien évident que les « retombées» seront multiples.

L'exploitation des données accumulées permettra de déterminer les facteurs défavorables ou limitants dans chaque type de sol et de supputer des chances d'intervention.

Nous pourrons en particulier nous appuyer sur les résultats de l'étude productionnutrition pour implanter un réseau d'essai de fertilisation rationnel, ou un réseau d'essai de techniques culturales lorsque ce seront les propriétés physiques qui seront limitantes.

D'autre part, il est bien évident que cette étude globale fera apparaître de nom- 
breux points qui mériteront d'être approfondis et qui pourront être le point de départ de nouveaux programmes de recherches.

Ces nouvelles voies de travail seront de deux sortes:

- L'une a trait à l'amélioration des techniques de mesure des caractéristiques de sols. On peut par exemple s’apercevoir que les techniques de dosage de phosphore sont inadéquates et ne correspondent guère à ce que l'arbre a absorbé. On sera donc amené à comparer diverses méthodes de façon à ne retenir que celle qui sera la plus adaptée dans ce cas particulier. On pourra même être amené à utiliser des méthodes d'extraction entièrement nouvelles.

- L'autre voie de travail a trait à des travaux plus fondamentaux, mais qui deviennent indispensables si l'on veut aller plus loin dans l'explication du comporte ment d'un végétal vis-à-vis des facteurs du milieu.

On sera par exemple amené à assayer de savoir pourquoi des troubles de la nutrition azotée se produisent dans certains milieux (BonneAu, 1967). Ces troubles pourront provenir d'une insuffisance de production azotée du sol, ou d'une période de production concordant mal avec la période de besoin maximal de l'arbre, ou d'une inaptitude du végétal à assimiler telle ou telle forme d'azote. On se tournera donc vers des procédés d'investigation assez fins et de caractère assez fondamental.

Dans un autre ordre d'idées, les possibilités de déterminer valablement les liaisons entre caractéristiques du sol et caractéristiques de nutrition dépendent de certaines connaissances de la physiologie de l'arbre.

L'acquisition de ces connaissances physiologiques se justifie par divers besoins:

- compréhension des anomalies apparentes des analyses foliaires, anomalies liées aux phénomènes de dilution de certains éléments minéraux dans la masse foliaire ;

- approche des phénomènes d'interaction entre alimentation en eau et alimentation minérale ;

- affinement du diagnostic foliaire par la prise en considération, non plus seulement des éléments totaux, mais de leurs diverses formes d'organisation ;

- interaction entre absorption des divers éléments, etc.

La méconnaissance de ces facteurs peut conduire à des erreurs assez grossières sur la disponibilité d'un élément dans le sol.

\section{VII. - DIFFICULTES D'UNE TELLE ETUDE}

Indépendamment des diverses difficultés que nous avons soulevées dans les paragraphes précédents et qui ont trait essentiellement aux mesures des facteurs du milieu, deux grandes autres séries de difficultés se présentent pour la réalisation d'une telle étude.

Ce sont les variations souvent aléatoires des facteurs pédologiques en fonction de la profondeur du sol, et les variations de production d'ordre génétique.

Enfin, dans le cas d'essences très récemment introduites, un tel type d'étude est plus difficilement réaiisable. 


\section{Variations aes facteurs pédologiques en fonction de la profondeur du sol}

Une très grande difficulté se présente lorsqu'il s'agit de relier les caractéristiques du sol à une variable qui en dépend comme la production.

En effet, un sol forestier est un ensemble hétérogène dont les propriétés varient considérablement en fonction de la profondeur.

Pour évaluer par exemple la richesse d'un sol en phosphore, il faudra déterminer la teneur en cet élément des divers horizons. On obtiendra non pas une valeur, mais un ensemble de valeurs exprimant la variation de la teneur en phosphore en fonction de la profondeur.

Quelle valeur retenir pour exprimer ou évaluer la teneur en phosphore de l'ensemble du profil ? Il est bien certain qu'une moyenne n'aura qu'une signification assez grossière.

D'autre part, si l'on retient tous les chiffres, il devient extrêmement difficile d'effectuer une interprétation.

En effet, les horizons étant variables d'un sol à l'autre, aussi bien par leur nature, leur nombre, leur épaisseur, il est pratiquement impossible d'effectuer des prélèvements à profondeur constante.

Nous pouvons opter pour la solution suivante: prélèvements effectués dans tous les horizons.

Les résultats sont ensuite exprimés par volume de sol et par tranche successive égale de sol. Les calculs sont effectués en tenant compte de la densité apparente de l'épaisseur et de la teneur en cailloux de chaque horizon. Nous pouvons ainsi obtenir une série de chiffres comparables pour tous les profils. Nous aurons par exemple la teneur en phosphore des 25 premiers centimètres, de 25 à 50 , de 50 à 75 , de 75 à $100 \mathrm{~cm}$, et la teneur totale du volume de sol prospecté par les racines.

Par ce procédé, il devient possible d’effectuer un travail d'interprétation assez facilement.

Le problème reste cependant pour les variables dont il n'est pas possible d'effectuer de sommations, comme par exemple le $\mathrm{pH}$ ou la densité apparente.

Pour ce type de caractéristiques, si la variation s'effectue avec la profondeur toujours dans le même sens, en augmentant ou en diminuant, on peut calculer des profondeurs pour lesquelles ces variabies prennent des valeurs données. On obtient ainsi des séries de paramètres parfaitement comparables d'un profil à l'autre.

$\mathrm{Si}$ les variations sont aléatoires en fonction de la profondeur, le problème est plus difficile, mais non insoluble.

Nous voyons que ces variations des caractéristiques du sol en fonction de la profondeur compliquent singulièrement ce genre d'étude, mais il semble toujours possible de trouver une solution.

\section{Variations de production dues à des facteurs d'ordre génétique}

Ce problème est certainement un des plus graves qui puissent se présenter pour ce genre d'étude.

La production dépend, en effet, non seulement de facteurs climatiques et pédologiques, mais également de facteurs génétiques. A l'intérieur d'une essence ou d'une espèce définie par certaines caractéristiques morphologiques, il existe des races ou 
provenances dont les caractéristiques physiologiques propres varient à l'intérieur des caractéristiques définissant l'espèce.

La réaction d'une essence à certains facteurs du milieu variera donc suivant les races considérées.

Une part de la variation de production observée aura donc une origine génétique, et sera par conséquent due à des facteurs impossibles à mesurer ou à chiffrer d'une manière quelconque.

Seule l'expérimentation poursuivie sous forme de plantations comparatives de provenances, par la Station d'Amélioration des Arbres forestiers, permet d'évaluer l'amplitude de la variabilité génétique d'une espèce. Il est possible de pousser plus loin l'analyse et d'évaluer l'interaction provenance station, grâce à des plantations comparatives identiques établies dans des stations différant par leurs conditions écologiques (J.-F. Lacaze).

L'interprétation que l'on pourra faire de l'action des facteurs du milieu pourra donc en être fortement perturbée, et cela d'autant plus que la variation génétique sera importante.

Pour les essences naturelles, il est probable que le danger est assez minime, surtout si l'on travaille par zone climatiquement homogène.

Pour le Peuplier, le problème ne se pose pas, puisqu'il est possible de déterminer le clône à partir de données morphologiques ou physiologiques simples (date de floraison, etc.). Par contre, pour les essences résineuses introduites, nous risquons fort de perdre une bonne partie de l'information et de n'obtenir que des fourchettes assez lâches de prévisions de la production.

Dans certains cas exceptionnels, il est possible de travailler sur des origines assez bien définies (Pin maritime dans les Landes de Gascogne) ; malheureusement, dans la majorité des cas, le problème est insoluble, au moins dans un avenir proche.

\section{Essences très récemment introduites}

Nous terminerons ce paragraphe en mentionnant le cas des essences très récemment introduites. Il est évident que la plupart du temps ces essences ne constituent que de jeunes peuplements ne couvrant pas toujours toute la gamme des stations de la région que l'on veut étudier.

Il est d'autre part plus difficile d'avoir un indice de production avec de jeunes peuplements qu'avec des peuplements plus âgés, et d'autre part, dans les premières années, certains facteurs difficilement chiffrables ont une action majeure, comme par exemple la concurrence herbacée, la concurrence de la strate arbustive ou la méthode de plantation.

Les renseignements que l'on pourra obtenir dans ces conditions seront évidemment beaucoup moins complets, surtout si le nombre de peuplements est insuffisant.

\section{CONCLUSIONS}

Nous constatons qu'actuellement nous connaissons extrêmement mal l'action des facteurs du milieu, et en particulier l'action des facteurs de sol sur la production des principales essences forestières, comme d'ailleurs sur la qualité technologique du bois. 
Il est indispensable que dans un avenir aussi proche que possible ces connaissances, qui sont essentielles pour les praticiens, soient acquises.

Nous proposons une méthode générale de travail basée sur l'enquête et qui doit être applicable à la plupart de nos grandes essences comme à la plupart des grandes régions forestières. Cette méthode est directement inspirée des très nombreux travaux effectués sur ce sujet depuis une cinquantaine d'années.

Cette méthode consiste à choisir un certain nombre de points d'échantillonnage, de manière à couvrir la gamme complète de sols ou de stations pour une région climatiquement homogène. Cette condition d'homogénéité n'est d'ailleurs pas requise quand on peut facilement tenir compte des variations climatiques.

En chaque point, nous effectuons trois séries de mesures : la mesure des caractéristiques de l'essence étudiée (production, composantes de la production, caractéristiques technologiques), la mesure des caractéristiques du milieu, et la mesure par analyses foliaires des caractéristiques de nutrition, qui sont la résultante de l'action de certains facteurs du milieu et de mécanismes physiologiques.

En outre, chaque fois que cela sera possible, on aura avantage à effectuer des relevés floristiques susceptibles de fournir des critères de détermination simple des types de stations.

A partir de ces diverses données, nous pouvons essayer de dépouiller l'action des divers facteurs du milieu, soit par des procédés simples faisant appel à des notions synthétiques regroupant tout un ensemble de facteurs, comme par exemple le type génétique et la roche-mère, soit par des procédés beaucoun plus complexes, essentiellement analyses statistiques multivariates, dont l'emploi en France a été introduit en matière forestière par Tomassone et MiLlier.

Par ces divers procédés, nous sommes susceptibles d'apporter les résultats suivants :

$1^{\circ}$ détermination des facteurs du milieu qui agissent sur la croissance ou toutes autres caractéristiques des diverses essences d'une région;

2" prévision de la production en fonction de conditions données de milieu et contribution à une politique de planification forestière régionale ;

3 amélioration des connaisances sur la physiologie de la nutrition minérale et de l'alimentation en eau ;

$4^{\circ}$ détermination des liaisons entre facteurs du milieu et nutrition;

$5^{\circ}$ mise au point de techniques d'amélioration des conditions de milieu.

Un outil extrêmement efficace pourra ainsi être donné aux sylviculteurs de terrain. De plus, un tei travail nous apportera un ensemble de connaissances qui nous permettront d'asseoir des systèmes d'expérimentation en vue de l'amélioration des propriétés du sol sur des bases rationnelles.

Enfin, les résultats qui pourront ainsi être obtenus par ce type d'enquête pourront être le point de départ d'une multitude de travaux de recherches beaucoup plus fins, concernant des points particuliers reiatifs aussi bien aux caractéristiques du milieu qu'aux caractéristiques physiologiques des arbres.

Nous avons énuméré les difficultés que l'on pouvait rencontrer. Elles sont multiples, et si nous espérons pouvoir fournir des réponses à beaucoup de questions, nous 
pensons aussi ne pas résoudre certains problèmes et faire apparaître de nombreuses questions nouvelles auxquelles il faudra ensuite répondre.

Pour mener à bien cie telles études, la collaboration de la plupart des stations du C.N.R.F. est indispensable.

Ce type d'étude nécessite donc, plus que tout autre, un programme d'action concertée au sein du C.N.R.F. en fonction des orientations que désire prendre chaque station concernée, et en fonction des besoins qui devraient être définis par les responsables de la politique forestière française.

Reçu pour publication en août 1970.

\section{SUMMARY}

REFLECTIONS UPON A METHOD FOR STUDYING RELATIONS BETWEEN ENVIRONMENTAL. FACTORS AND FOREST STAND CHARACTERISTICS

We note that nowadays, our knowledge of the effect of environmental factors and particularly of soil factors upon the yield of the principal forest species, as well as upon the technological quality of wood, is quite vague.

This knowledge, which is quite essential for foresters, must be acquired as soon as possible.

We propose a general method of study based upon investigation and which is probably appliable to most of our forest species and to most of our forest regions. This method results directly from the last fifty years numerous studies.

The method consists in choosing a certain number of sampling places so that every kind of soil or site is represented, within a homogeneous climatic region. Yet this homogeneous quality is not required if we can easily record the climatic variabilities. On each place of sampling, we select three kinds of measures: measure of the characteristics of the choosen species (yield, yield components, technological characteristics, etc...) measure of the environmental characteristics and measure, by foliar analysis of nutrition characteristics, which are the resultant of some environmental factors and of physiological mecanisms.

Besides, when possible, it is best to study forest flora which may give some interesting indications about the sites.

From these different indications, we can try and analyse the action of the various environmental factors, either by simple methods referring to synthetic notions for example the genetic type of soil and the parent rock, either by far more complex proceedings, essentially multivariate statistical analyses (introduced in forest matter in France by Tomassone and Millier).

From these different methods, we are able to obtain the following results:

$1^{\circ}$ Determination of environmental factors which have influence upon the growth or any other characteristic of the different species of a region.

$2^{\circ}$ Forecast of the yield according to the given environmental factors and contribution to a policy of regional forest planification.

$3^{\circ}$ Improvement of our knowledge of the physiology of mineral nutrition and water alimentation.

$4^{\circ}$ Determination of the relations between environmental factors and nutrition.

$5^{\circ}$ Setting up of a method in order to improve environmental conditions.

So a very efficient implement will be given to foresters. Moreover such a work will bring us a whole knowledge which will permit us to give rational bases to experimental systems in order to improve soil qualities.

The results obtained by this kind of research can be the very beginning of a lot of far more detailed researches concerning precise problems referring to environmental factors or to physiological characteristics of trees.

We have mentioned the difficulties which can be met. They are numerous and if we 
hope to be able to give answer to many questions, we also think that we shall not be able to resolve some problems and that we shall raise numerous new questions to which we shall then have to answer.

To carry through such researches, the collaboratories of most of C.N.R.F. laboratories is necessary.

This kind of research needs, more than any other one, a program of concerted actions within the C.N.R.F., according to the orientations wanted by every concerned laboratory, and according to the needs which should be clearly defined by the heads of french forest policy.

\section{ZUSAMMENFASSUNG}

\section{METHODISCHE ÜBERLEGUNGEN ZUR UNTERSUCHUNG DER BEZIEHUNGEN ZWISCHEN} STANDORTFAKTOREN UND BESTANDESMERKMALEN

In der vorliegenden Arbeit wird eine allgemeine Arbeitsmethode zur Untersuchung des Einflusses der Umweltfaktoren, insbesondere des Bodens, auf die Wuchsleistung oder andere Bestandesmerkmale dargestellt, wobei den seit mahezu fünfzig Jahren erschienen überaus zahlreichen Arbeiten auf diesem Gebiet Rechnung getragen wird.

Die Methode kann kurz wie folgt zusammengefasst werden :

- Innerhalb eines klimatisch soweit als möglich einheitlichen Gebietes werden eine gewisse Anzahl von Probebeständen ausgewählt welche sich über sämtliche vorkommeneden Boden - oder Standorttypen erstrecken.

- In jedem Probebestand werden die verschiedenen Bestandesmerkmale, der Ernährungszustand und die verschiedenen Standortfaktoren insbesondere die Bodenmerkmale, gemessen und beschrieben.

Bei der Auswertung wird versucht die zwischen diesen drei Datengruppen bestehenden Beziehungen darzustellen, wobei zwei Wege begangen werden können:

- Eine sehr einfache graphische oder statistische Methode untersucht entweder die Beziehungen zwischen Einzelfaktoren denen jedoch eine gewisse Bedeutung begeméssén wérden kann, oder zwischen synthetischen Merkmalen wie z. B. Bodentyp oder Grundgestein.

- Auswertung mit verschiedenen multivariaten Analysenmethoden.

Die Ergebnisse dieser Arbeitsmethode erlauben eine Wuchsleistungsschätzung als Funktion der Umweltfaktoren, vermitteln eine solide Grundlage für die Anlage von Versuchen zur Standortverbesserung und können gleichfalls als Ausgangsbasis für spezielle Detailuntersuchungen verwendet werden. Eine enge Zusammenarbeit zwischen den verschieden forstlichen Fachgebieten erweisst sich als unumgänglich notwendig.

\section{REFERENCES BIBLIOGRAPHIQUES}

Aussenac G., Becker M., 1968. Ecologie d'un massif sur sols hydromornhes. La forêt de Charmes (Vosges). Contribution à la mise au point d'une méthode d'étude dynamique du milieu forestier. Ann. Sci. forest. (4).

Becker M., 1968. Le hêtre et ses problèmes en forêt de Villers-Cotterets. Contribution à la mise au point d'une méthode dynamique d'étude écologique du milieu forestier. Publication du Laboratoire de Botanique forestière, C.N.R.F., Nancy.

Bonneau M., Gelpe, Le Tacon F., 1968. Influence des conditions de nutrition minérale dans le dépérissement du pin maritime dans les Landes de Gascogne. Ann. Sci. forest., 25 (4).

Carmean W. H., 1954. Site quality for Douglas Fir in Southwestern Washington and its relationship to precipitation elevation, and physical soil properties. Proc. Soil Sci. Soc. Amer, 18, 330334.

CoILE T.S., 1938. Classification of forest sites with special reference to ground vegetation. Journ. For., 36, 1062-1066.

CoIle T.S., 1948. Relation of soil characteristics to site index of loblolly and shortleaf pines in the lower Piedmont region of North Carolina Duke Univ. School of Forestry, Bull., 13, 78 pp. 
Coile T. S., Schumacker F. X., 1953. Relation of soil properties to site index of loblolly and shortleaf pines in the Piedmont region of the Carolinas, Georgia and Alabama. Journ. For., 51, 739-744.

DAGNÉLIE D., 1956-1957. Recherches sur la productivité des hêtraies d'Ardennes en relation avec les types phytosociologiques et les facteurs écologiques. Bull. Inst. Agron. Sta. Rech. Gembloux, 24, 249-284, 25, 44-94.

Donahue R. L., 1940. Forest site quality studies in the Adirondacks: I. Tree growth as re'ated to soil and morphology. Cornell. Univ. Agric. Expt. Sta. Mem., 229, 44 pp.

Duchaufour, Alt, 1958. Un exemple d'utilisation pratique de la cartographie des stations: la forêt du Ban d'Etival (Vosges). R.F.F., 10, 597-630.

Decourt N., 1965. Le pin sylvestre et le pin laricio de Corse en Sologne. Tables de production provisoires et méthodes utilisées pour les construire. Ann. Sci. Forest., 22 (2), 259-318.

Decourt N., 1967. Le Douglas dans le Nord-Est du Massif Central. Tables de production provisoires. Ann. Sci. for., 24 (1), 45-84.

Decourt N., Godron M., Romane F., Tomassone R., 1969. Comparaison de diverses méthodes d'interprétation statistique de liaison entre le milieu et la production du pin sylvestre en Sologne. Ann. Sci. For., 26 (4), 413-443.

DeCourT, 1966. Instruction pour l'assiette et les mensurations de placettes temporaires et semi-permanentes. Publication ronéotypée de la Station de Sylviculture et Production.

GaIser R. N., 1950. Relation between soil characteristics and site index of Lobloly Pine in the coastal plain region of Virginia and the Carolina. Journ. For., 48, 271-275.

Gessel S. P., 1949. Correlation between certain soil characteristics and site for Douglas Fir in northwestern Washington. Proc. Soil. Sci. Soc. Amer., 14, 333-337.

GESSEL S. P., Lloyd W. J., 1950. Effect of some physical soil properties on Douglas Fir si.e quality. Journ. For., 48, 405-410.

Geborkiantz S. R., Scholz H. F., 1944. Determining site quality in understocked oak forests. Journ. For., 42, 808-811.

Hartmann F. K., 1926. Die Abhängigkeit der Höhenbonität und der Bodenflora der Kiefer vom Feinerdegehalt und Untergrund gewisser diluvialer Sans-Böden. Zeitschr. Forst u Jagdw, 58 , 226-255.

Ilvessalo Y., 1923. Ein Beitrag zur Frage der Korrelation zwischen den Eigenschaften des Bodens und dem Zuwachs des Waldbestendes. Acta Forest Fenn., 25, 31 pp.

Jones John R. ,1969. Review and comparison of site evaluation methods. USDA Forest ${ }^{\mathrm{e} S e r v i c e}$ Research Paper RM-SI, 27 pp. Rocky Mountain Forest and Range Experiment Station, Fort Collins, Colorado 80521.

Korstian C. F., 1917. The indicator significance of native vegetation in the determination fo forest sites. Plant World, 20, 267-287.

Korstian C.F., 1919. Native vegetation as a criterion of site. Plant World, 22, 253-261.

LaCaze J.-F., 1970. Analyse d'une expérience multistationnelle de provenances d'épicéa. Ann. sci. forest. (1).

Leroy Ph., 1967. Variations de productivité du chêne sur les pentes à Bercé. Nécessité d'une cartographie des sols. R.F.F., $\mathrm{n}^{\circ} 10$.

LEROY Ph., 1968. Etudes de nutrition production sur le chêne, perspectives de fertilisation. R.F.F., $\mathrm{n}^{\circ} 5$.

LE TACon F., 1968. Un essai d'application de la cartographie des sols. Nécessité de la connaissance des facteurs de production. R.F.F., $\mathrm{n}^{\circ} 4$.

Le Tacon F., Oswald H., 1969. Les sols en Haute-Ardèche. Caractérisation et production forestière. Bulletin Association française pour l'étude du sol (en cours d'impression).

LÉvy G., 1967. Influence de certaines propriétés du sol sur la densité d'enracinement de l'épicéa commun et du pin sylvestre. Thèse Docteur-Ingénieur, Faculté des Sciences, Nancy.

Lowry G. L., 1964. Forest Soil Sie Studies I. Objectives, Sampling, preliminary results on black spruce P.P.R.I.C. Technical reports, $\mathrm{n}^{\circ} 368$.

LuNT H. A., 1939. Soil characteristics topography and lesser vegetation in relation to site quality of second-growth oak stands in Connecticut. Journ. agr. Res., 59, 407-428.

Myers C. A., Deasen J. L. van, 1960. Site index of Ponderosa Pine in the Balck Hills from Soil and topography. J. For., 58, (7).

Oswald H., 1969. Les potentialités forestières en Haute-Ardèche, l'épicéa commun (Picea abies L.).

PARDE J., 1961. Dendrométrie, 360 p. Imprimerie Louis-Jean, Gap.

RENNIE P. J., 1963. Methods of assossing Forest site capacity. Commonwealth forestry Review, vol. 42, (4), $\mathrm{n}^{\circ} 114$, décembre 1963.

RIGG G. B., 1929. Using the vegetation cover as an aid in studying logged-off lands as forest sites. Journ. For., 27, 539-545. 
Rowe J. P., 1956. Classification of site for forest management Int. Union of forest. Res. Org. 12th Congr. Oxford.

Row C., 1960. Soil site relations of old fuld, Slash Pine plantations in Carolina sans hills. F. For., 58, (9).

Spils Bulri R. H., Smith D. A., 1957. Forest site of the pacific Northwest Canada. Ministère des Terres et Forêts. Service forestier.

Stoeckeler J. H., 1948. The growth of Quaking Aspen as affected by soil properties and fire. Journ. For., 46, 727-737.

Tamm O., Wadman E., 1945. Sur les facteurs stationnels et leur corrélation avec les conditions forestières dans le district de Hamra (Texte suédois), Svenska Skogs., Tidskr., Bihang, 2 , 79 pp.

TARRAnT R. F., 1949. Douglas-Fir site quality and soil fertility. Journ. For., 47, 716-720.

Timbal J., 1968. Contribution à l'étude des associations forestières de la forêt de Ste-Hélène. Vosges. Thèse de $3^{\mathbf{e}}$ cycle. Fasc. Sci. Orsay.

Tomassone R., 1965. L'analyse des composantes principales. Publication ronéotypée de la station de Biométrie. C.N.R.F. Nancy.

Tomassone R., 1965. Analyse de régression progressive. Publication ronéotypée de la station de Biométrie. C.N.R.F. Nancy.

Tomassone R., 1967. Régression multiple progressive. Station de Biométrie. C.N.R.F. Nancy Programme A. G. 65007.

Tomassone R., 1967. Une méthode d'investigation; la régression orthogonale. Ann. Sci. forest., 24, (3), 233-258.

VAlléE G., 1967. Utilisation des relations entre la végétation du sous-bois et la productivité des stations pour la classification des forêts. Thèse Doct. Ing. $2^{\mathrm{e}}$ sujet. Fasc. Sciences de Nancy.

Viro P. J., 1947. La composition granulométrique et la fertilité des sols forestiers en considérant principalement la pierrosité (Texte finlandais). Commun. For. Fenn., 35, 115.

Youngberg C. T., Scholz H. F., 1949. Relation of soil fertility and rate of growth of mixed oak stands in the Driftless area of Southwestern Wisconsin. Proc. Soil. Sci. Soc. Amer., 14, 331-332. 